\title{
Analysis of dose distribution between contemporary and standard planning in high-dose-rate endobronchial brachytherapy based on three- dimensional imaging
}

\author{
Marcin Sawicki, MSc, MD, PhD',2, Jarosław Łyczek, MD, PhD², Prof. Zbigniew Szutkowski, MD, PhD³ \\ 'Faculty of Medicine, University of Rzeszów, Poland, 2Department of Brachytherapy, Subcarpathian Cancer Center, Brzozów, Poland, \\ ${ }^{3}$ Department of Radiotherapy, Maria Skłodowska-Curie Memorial Cancer Center and Institute of Oncology, Warsaw, Poland
}

\begin{abstract}
Purpose: The treatment planning (TP) in high-dose-rate (HDR) endobronchial brachytherapy (EB) can be based on various forms of imaging. In the case of lung cancer, one-dimensional or two-dimensional imaging is standard. The dose coverage of the target (planning target volume - PTV) and organs at risk (OAR) is unknown, because the doses are calculated on the basis of the dose points. In modern brachytherapy, TP can be based on three-dimensional (3D) images. A plan created in this way contains information about the dose distribution in the PTV and OAR. Treatment plans based on standard planning (SP) and contemporary planning $(\mathrm{CP})$ may differ in dose distribution in the patient's body. Those differences between SP and CP may have an effect on the dose distribution in PTV, OAR and follow-up.

Material and methods: The study involved a group of 31 patients prospectively treated with advanced, inoperable, non-small cell lung cancer. As many as 76 treatment fractions were analyzed. Firstly, the coverage of the PTV parameter in 2D and 3D for $V_{85}, V_{100}$ and $V_{115}$ was analyzed. Secondly, the dosage that OAR would take in was evaluated. In the cases of the heart, spinal cord and esophagus, the examined dosage equaled $D_{0.1 \mathrm{~cm}^{3}}, \mathrm{D}_{1 \mathrm{~cm}^{3}}$ and $\mathrm{D}_{2 \mathrm{~cm}^{3}}$ for each of the structures. Also, heart $\mathrm{D}_{20}$ was examined as well as $\mathrm{D}_{5}$ for the healthy lung.

Results: The median dose to the target volume was on average $43.33 \%$ higher for $\mathrm{V}_{85}$ with the contemporary planning method when compared to standard planning, with statistical significance. This came with the cost of an OAR mean dose increase of $1 \mathrm{~Gy}$ in $\mathrm{D}_{0.1 \mathrm{~cm}^{3}}$ for the heart.

Conclusions: Contemporary TP in EB allows one to adjust the dose distribution for individual clinical situations and allows one to improve clinical target volume (CTV) coverage, increase doses to the OAR and increase overall survival. The use of new methods of treatment plans in EB has significantly increased the follow-up to 21 months in a treated group of patients.

J Contemp Brachytherapy 2019; 11, 5: 462-468 DOI: https://doi.org/10.5114/jcb.2019.89194
\end{abstract}

Key words: endobronchial brachytherapy, treatment planning, three-dimensional imaging.

\section{Purpose}

Lung cancer is one of the most prevalent cancers worldwide. It can be divided into two pathomorphological forms: non-small cell (80\%) and small cell (20\%) carcinomas $[1,2,3,4,5]$. In patients with less advanced cancer, the main treatment modality is radical surgery. Patients who refuse surgery or whose general health does not allow surgery are treated with radical radiotherapy and/ or chemotherapy [6]. In the case of a patient at a late clinical stage, the cancer is permanently inoperable. The treatment method in such cases relies on alleviating the symptoms of the disease and extending the survival time. Patients are treated with palliative radiotherapy. In palliative radiotherapy, the most common treatment is brachytherapy $[7,8,9]$. HDR brachytherapy is the most frequently used method of treating the symptoms of inoperable lung cancer in advanced form, in which the tumor may narrow or obstruct the bronchial tree, resulting in symptoms such as hemoptysis, dyspnea, cough and lung inflammation. Endobronchial brachytherapy (EB) is an indication mainly for palliative treatment $[10,11,12,13]$. The universality of high-dose-rate (HDR) brachytherapy in the area of the lung is mainly due to the use of automatic remote afterloaders, a stepping source, flexible applicators and a short treatment time 
$[10,14,15]$. Treatment planning (TP) in HDR EB can be based on various forms of imaging. One or multiple-dimensional images can be applied. In the case of lung cancer, 1D (imaging from one angle) or 2D (view from two planes) imaging is standard. In the case of one-dimensional planning systems, the dose distribution is presented in virtual form along the unreal, rectilinear applicator. Neither the anatomical conditions of the patient nor the curvature of the applicator are included in the dose distribution. The treatment plan can also be based on $2 \mathrm{D}$ or $3 \mathrm{D}$ images. If the images are $2 \mathrm{D}$, then the curvature of the applicator is taken into account. 2D imaging does not provide complete information about the dose in the risk area or critical organs [16]. Using this type of imaging, the dose is normally specified by dose points [17]. The dose points are at a constant distance from the axis of the applicators. One of the limitations of imaging is that the dose distribution at target and organs at risk (OAR) are unknown. In modern brachytherapy, a computerized $\mathrm{TP}$ system allows the use of 3D images. A plan created in this way contains information about the dose distribution in the planning target volume (PTV) and OAR. Knowledge about the dose distribution allows the creation of an individual treatment plan for a particular patient $[18,19]$. Standard planning (SP) is realized on dose distribution at a distance of $1 \mathrm{~cm}$ from the applicator. The contemporary planning $(\mathrm{CP})$ in $\mathrm{EB}$ takes into account the dose distribution in real PTV and real OAR. Mostly, departments which are using 3D images adopt standard planning in 3D imaging. In that case, the dose distribution is still calculated $1 \mathrm{~cm}$ from the applicator and PTV is the volume at a distance of $1 \mathrm{~cm}$ from the applicator. Planning systems based on 3D imaging show the dose distribution in the volume of PTV and OAR and plans are adapted to the clinical situation. The aim of this study is to compare the dose distribution in TP based on SP and $\mathrm{CP}$ in EB. Based on this study, the real calculated doses in investigated OAR are to be presented.

\section{Material and methods}

The study involved a group of 31 patients prospectively treated with advanced, inoperable, non-small cell lung cancer. All patients were treated in the years 20112013. The age range of the study group was $52-85$ years (median 66). A total of 72 treatment fractions with 3D imaging were studied. In 46 fractions two separate applicators were used, while three applicators were used in nine treatments. One applicator was used in 21 cases. The prescription dose was 7 Gy per fraction. The number of fractions varied from 1 to 3 . For each treatment fraction, two treatment plans were created, independently for each planning method. They were prospectively planned to have SP and CP plans prepared, but all patients were treated using the $\mathrm{CP}$ method. Both planning methods were based on 3D images from a computed tomography (CT) scanner.

After the treatment, patients were scheduled for follow-up visits, during which the long-term treatment effects were assessed. To compare the observation of patients treated with the standard planning method, a group of 11 patients was analyzed. The patients who were treated by both methods historically had the same clinical qualifications. These were the patients who qualified only for palliative treatment due to the advanced clinical stage. TP was created in the Oncentra MasterPlan Brachy 4.0 system. Dose calculation algorithm TG-43 was used. The Lumencath Applicator Set was used for brachytherapy. The SPSS statistical package was used to perform the calculations. Student's $t$-test for two independent samples was used as it was the most appropriate for comparison of quantitative variables between two groups $(\alpha=0.05)$.

\section{Course of the procedure}

None of the treated patients required hospitalization. They participated in the procedure on an empty stomach. Patients underwent anesthetic consultations followed by premedication and initial CT scanning. The next step was videobronchofiberoscopy. After reaching the place where the cancerous area was located, or its immediate surroundings, a catheter was inserted through the videobronchofiberoscope. Depending on the location and extent of the disease in the place, the number of applicators ranged from 1 to 3 . Each applicator was equipped with a marker that allowed visualization of the catheter and its first active position in the imaging system. After the application, the patients underwent CT scanning, and if a physician successfully verified the application, the treatment planning process was continued.

\section{Contemporary planning (3D method)}

On imported CT images, the PTV area (volume of target dependent on the actual clinical situation) and OAR (heart, esophagus, lungs, spinal cord) were contoured. The next steps were: reconstruction of the applicator(s), activation of the source positions (source step size was $2.5 \mathrm{~mm}$ or $5.0 \mathrm{~mm}$ ) in the vicinity of the PTV. The next stage of the procedure was optimization of the dose distribution. Due to the placement of the implant in the patient's body, two optimization algorithms were used in the computer treatment planning system (TPS). Geometric optimization was used first, as it allows very high homogeneity between source stop points $[10,20,21]$. The final treatment plan was completed using graphical optimization. Graphical optimization consists of the manual adjustment of the isodoses, which illustrates the distribution of the dose in 3D images. This algorithm allows a unique dose distribution in PTV and OAR $[15,22]$.

\section{Standard planning (2D method)}

In the SP approach, the same 3D images and applicator/source positions were used as in the $\mathrm{CP}$ method, but the stopping times of the source were calculated using the dose point optimization method. Optimization to the points consists of fitting the reference isodose in such a way that the entire implant area is covered by the reference dose. When used to optimize the appropriate number of reference points, the dose distribution takes such a form that the planned dose is reached at each 
reference point $[20,23]$. Dose specification points were located at a distance of $1 \mathrm{~cm}$ from the applicator axis in a perpendicular direction and the active length was set to the length of the tumor with a margin of $0.5 \mathrm{~cm}$. Before dose optimization, dose normalization was performed at pre-determined reference points.

To the best understanding of differences in TP created by both methods, Figure 1 presents the 3D dose distribution for a standard and contemporary TP for the same patient and treatment fraction.

\section{Results}

In the analysis of dose distribution between contemporary and standard planning, PTV included the reference doses in the SP and CP method for $\mathrm{V}_{85}, \mathrm{~V}_{100}$ and $\mathrm{V}_{115}$. Then the doses that critical organs received in both methods were analyzed. In the case of the heart, spinal cord and esophagus, doses of $\mathrm{D}_{0.1 \mathrm{~cm}^{3}}, \mathrm{D}_{1 \mathrm{~cm}^{3},} \mathrm{D}_{2 \mathrm{~cm}^{3}}$ for each of the structures were calculated. $\mathrm{D}_{20}$ was also examined for the heart and lungs, and $D_{5}$ for healthy lung tissue. The two groups of variables were also compared by a box-and-whisker plot (interquartile range $-25^{\text {th }}-75^{\text {th }}$ percentile, "whisker" - irrelevant data range, "_" - me- dian, " $x$ " - average). The values of the whiskers on the box plot were determined by one and a half times the $1.5 \times \mathrm{IQR}$ quartile range.

\section{Reference dose in the target area}

Figure 2 presents box diagrams where the values for PTV $V_{85}$, PTV $V_{100}$, and PTV $V_{115}$ were compared in SP and CP methods. For PTV $V_{85}, V_{100}$ and $V_{115}$ median dose was increased by $43.33 \%, 44.08 \%$ and $43.67 \%$ with statistical significance $(p<0.001)$ for the CP method in relation to the SP method.

\section{Dose in the organ at risk - lung}

Figure 3 presents box diagrams where the values for the lung $\mathrm{D}_{20}$ and $\mathrm{D}_{5}$ for healthy lung tissue were compared using SP and CP methods. In the case of the lung $\mathrm{D}_{20}$ median dose $(\mathrm{SP}=0.96 \mathrm{~Gy} ; \mathrm{CP}=1.64 \mathrm{~Gy})$ was increased by 0.68 Gy (with statistical significance $p<0.001$ ) for the $\mathrm{CP}$ method in relation to the SP method. $\mathrm{D}_{5}$ for healthy lung tissue median dose $(S P=0.39$ Gy; $\mathrm{CP}=0.53 \mathrm{~Gy}$ ) was increased by $0.14 \mathrm{~Gy}$ with statistical significance $(p<0.01)$ for the CP method in comparison to the SP method.
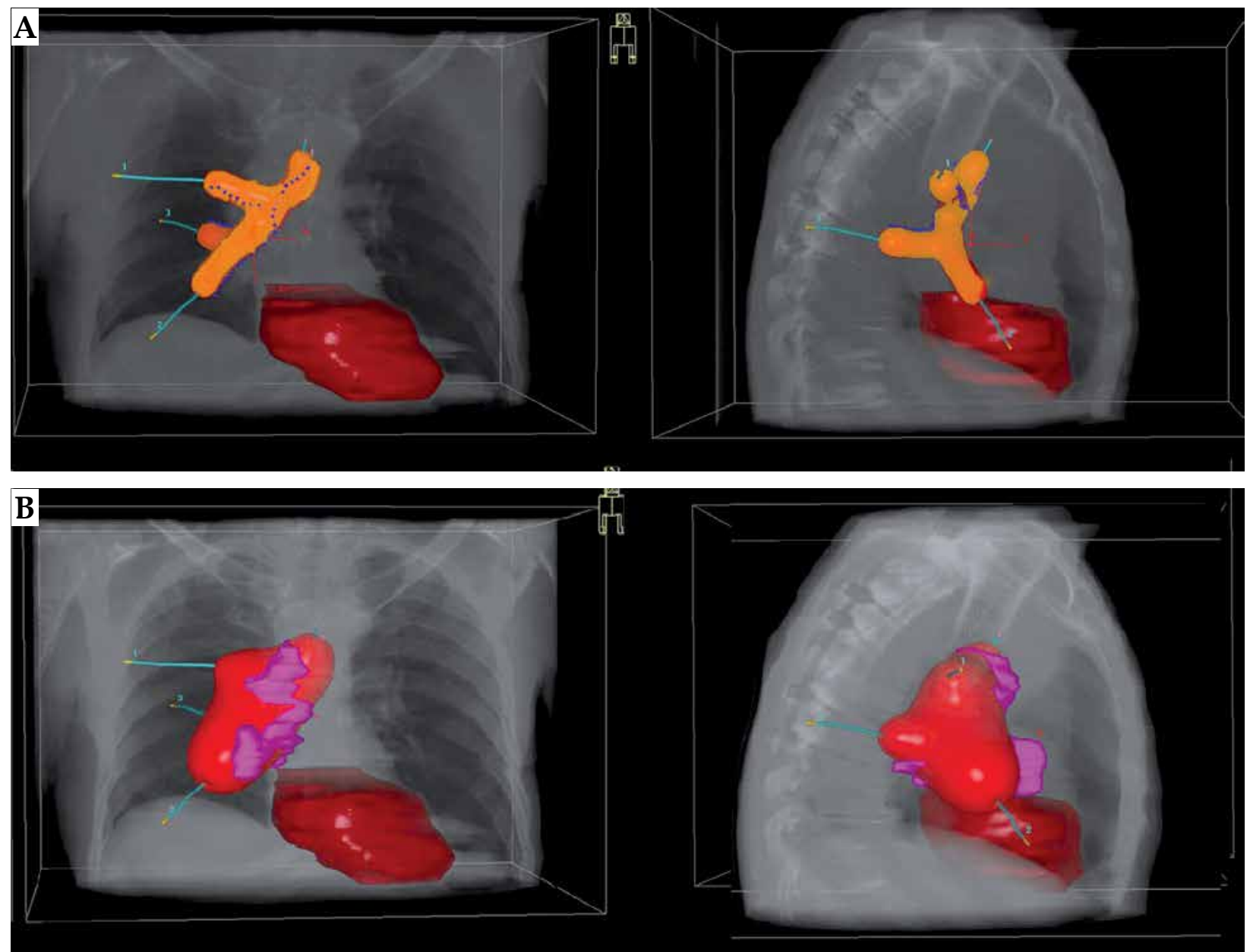

Fig. 1. Comparison of the distribution between SP $(\mathbf{A})$ and $\mathrm{CP}(\mathbf{B})$ method in regard to PTV volumes in relation to adjacent OAR. Prescribed $100 \%$ isodose $\left(\mathrm{V}_{100}\right)$ is presented in yellow for $\mathrm{SP}(\mathbf{A})$ and in red for $\mathrm{CP}(\mathbf{B})$ planning methods; PTV - purple; heart - dark red 


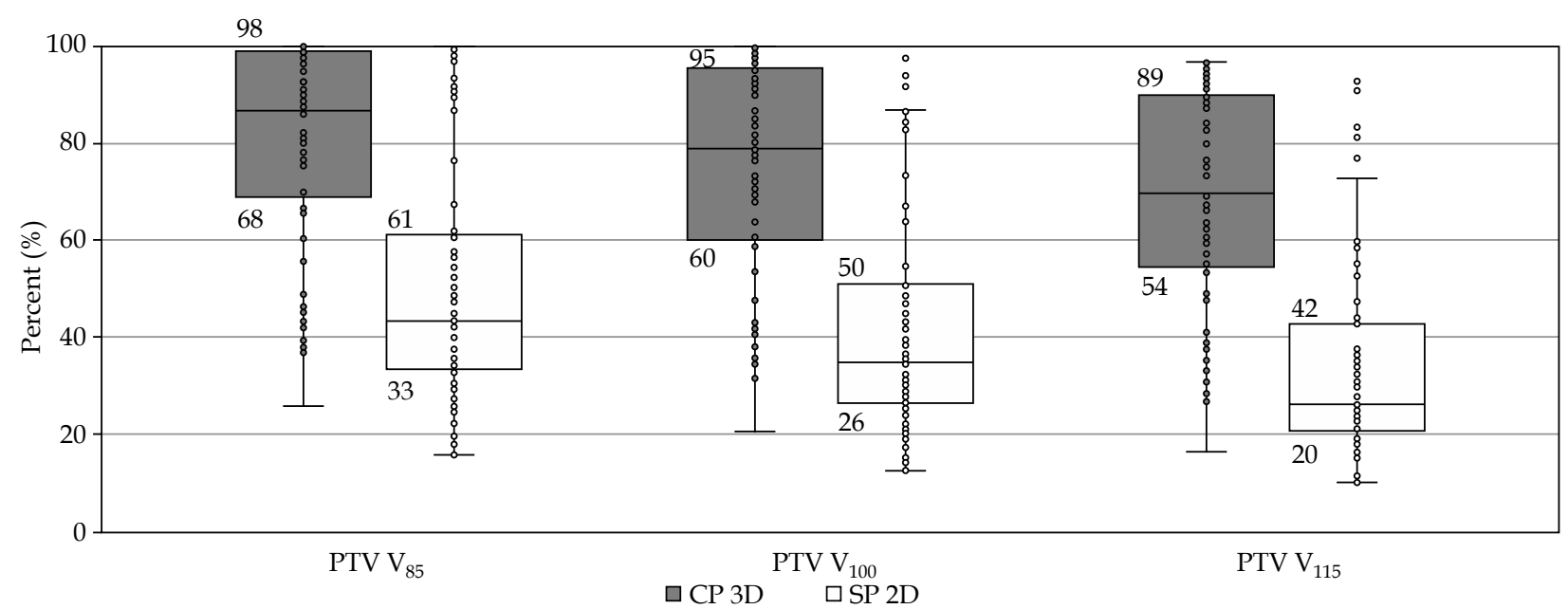

Fig. 2. Reference dose in the target area for contemporary and standard planning

\section{Dose in the organ at risk - esophagus}

Figure 4 presents box diagrams for the values of the esophagus $D_{0.1 \mathrm{~cm}^{3}}, D_{1 \mathrm{~cm}^{3}}, D_{2 \mathrm{~cm}^{3}}$. In the case of esophagus $\mathrm{D}_{0.1 \mathrm{~cm}^{3}}$, there were no statistically significant differences between the CP and SP methods ( $p=0.3)$. In the case of esophagus $\mathrm{D}_{1 \mathrm{~cm}^{3}}$, there were statistically significant differences between the $\mathrm{CP}$ and SP methods. However, as can be seen from the $p$-value below $0.05(p=0.02)$, these differences are small. In the case of esophagus $\mathrm{D}_{2 \mathrm{~cm}^{3}}$, median dose ( $\mathrm{SP}=1.64 \mathrm{~Gy} ; \mathrm{CP}=2.70 \mathrm{~Gy}$ ) was increased by 1.06 Gy with statistical significance $(p<0.001)$ for the CP method in relation to the SP method.

\section{Dose in the organ at risk - spinal cord}

Figure 5 presents box diagrams where the values for spinal cord $\mathrm{D}_{0.1 \mathrm{~cm}^{3}}, \mathrm{D}_{1 \mathrm{~cm}^{3}}$ and $\mathrm{D}_{2 \mathrm{~cm}^{3}}$ were compared be-

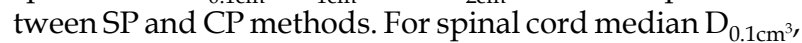
$\mathrm{D}_{1 \mathrm{~cm}^{3}}$, and $\mathrm{D}_{2 \mathrm{~cm}^{3}}$ was increased by $0.33 \mathrm{~Gy}(\mathrm{CP}=1.17 \mathrm{~Gy}$; $\mathrm{SP}=0.83 \mathrm{~Gy}), 0.34 \mathrm{~Gy}(\mathrm{CP}=0.93 \mathrm{~Gy} ; \mathrm{SP}=0.59 \mathrm{~Gy})$ and $0.32 \mathrm{~Gy}(\mathrm{CP}=0.85 \mathrm{~Gy} ; \mathrm{SP}=0.53 \mathrm{~Gy})$, respectively, with statistical significance $(p<0.001)$ for the $\mathrm{CP}$ method in relation to the SP method.

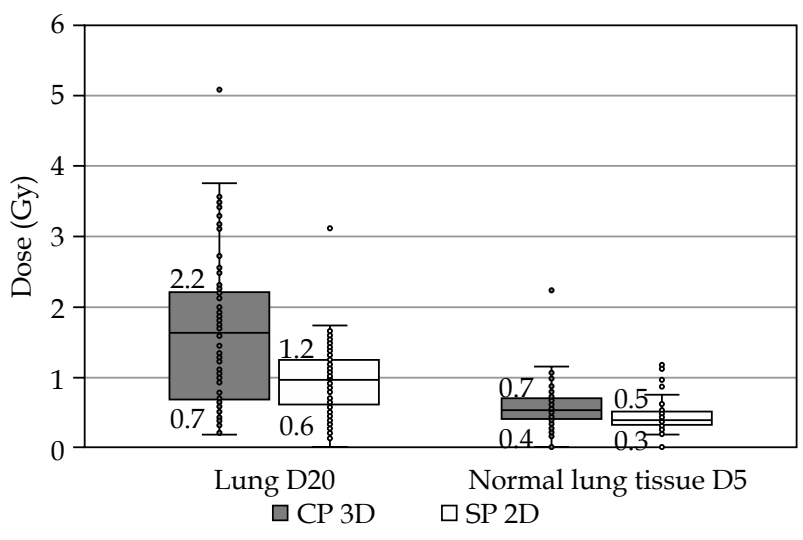

Fig. 3. Dose in the lung for contemporary $3 \mathrm{D}$ and standard 2D planning

\section{Dose in the organ at risk - heart}

Figure 6 presents box diagrams where the values for heart $\mathrm{D}_{0.1 \mathrm{~cm}^{3},}, \mathrm{D}_{1 \mathrm{~cm}^{3}}, \mathrm{D}_{2 \mathrm{~cm}^{3}}$ and $\mathrm{D}_{20}$ were compared between $\mathrm{SP}$ and $\mathrm{CP}$ methods. For heart $\mathrm{D}_{0.1 \mathrm{~cm}^{3}}, \mathrm{D}_{1 \mathrm{~cm}^{3}}, \mathrm{D}_{2 \mathrm{~cm}^{3}}$ and $\mathrm{D}_{20}$ median dose was respectively increased by $1 \mathrm{~Gy}$

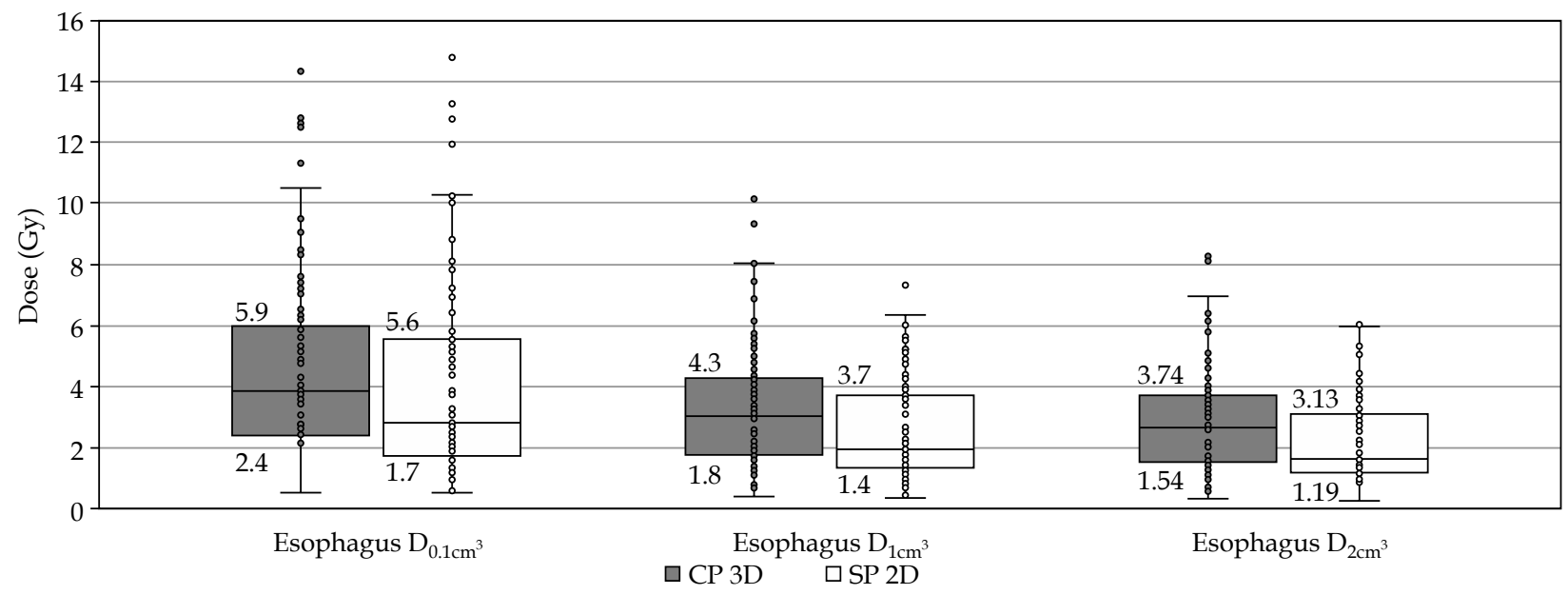

Fig. 4. Dose in the esophagus for contemporary 3D and standard 2D planning 


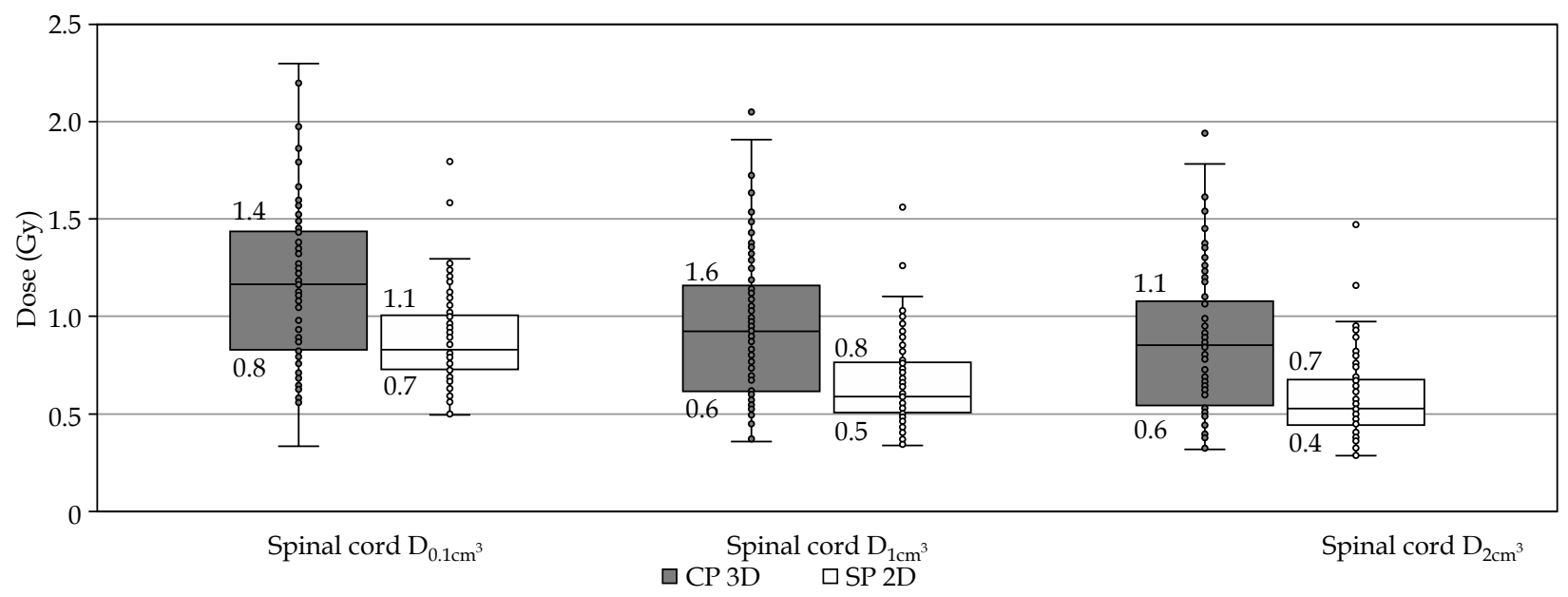

Fig. 5. Dose in the spinal cord for contemporary 3D and standard 2D planning

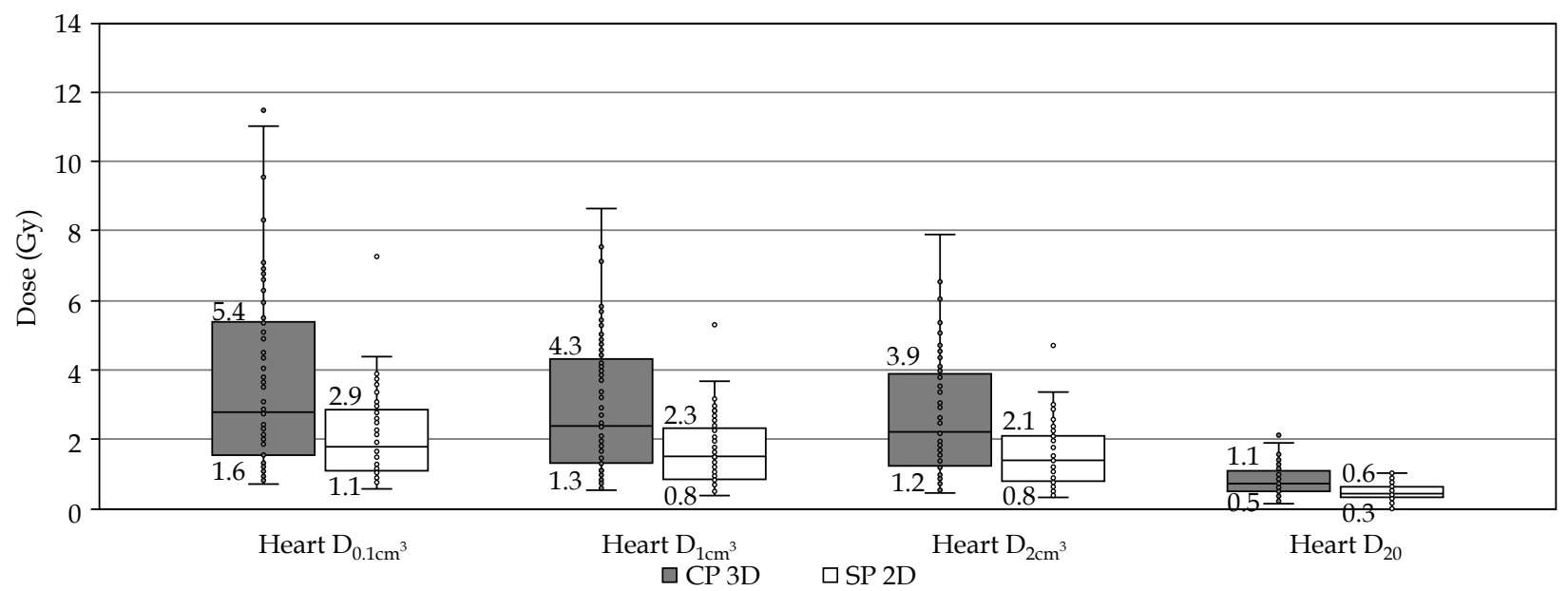

Fig. 6. Dose in the heart for contemporary 3D and standard 2D planning

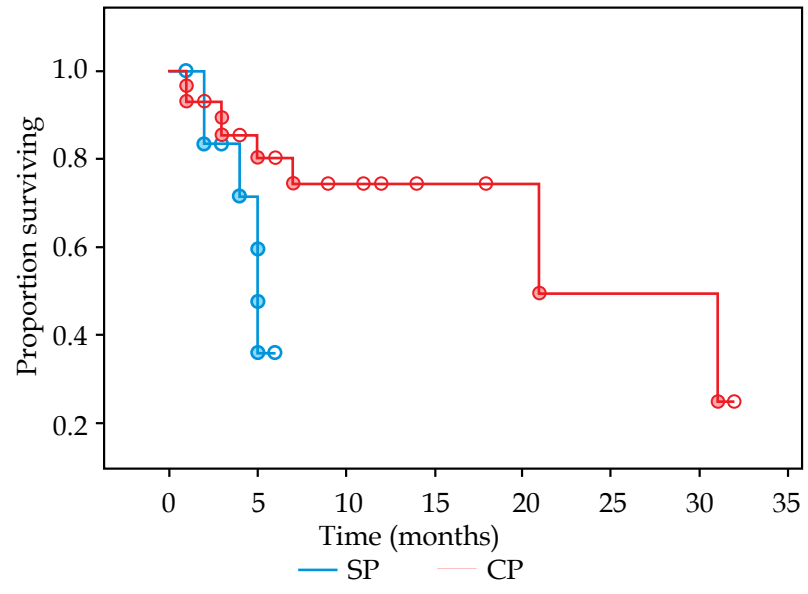

Fig. 7. Overall survival data for the 31 patients contemporarily treated with $\mathrm{CP} 3 \mathrm{D}$ method (in red) and for 11 patients historically treated with SP 2D method (in blue)

$(\mathrm{CP}=2.81 \mathrm{~Gy} ; \mathrm{SP}=1.81 \mathrm{~Gy}), 0.87 \mathrm{~Gy}(\mathrm{CP}=2.40 \mathrm{~Gy} ;$ $\mathrm{SP}=1.53 \mathrm{~Gy}), 0.81 \mathrm{~Gy}(\mathrm{CP}=2.22 \mathrm{~Gy} ; \mathrm{SP}=1.41 \mathrm{~Gy})$ and 0.29 Gy $(\mathrm{CP}=0.75 \mathrm{~Gy}$; $\mathrm{SP}=0.46 \mathrm{~Gy})$, with statistical sig- nificance $(p<0.001)$ for the CP method in comparison to the SP method.

\section{Analysis of survival and toxicity}

Figure 7 presents an analysis of survival time for patients after treatment. For patients treated with the $\mathrm{CP}$ method, the median duration of survival after treatment was 21 months. No late complications due to HDR-EB were observed in any patient treated with the $\mathrm{CP}$ method. Doses received by organs and critical structures in the $\mathrm{CP}$ method did not affect the toxicity of treatment.

\section{Discussion}

One of the methods employed to reduce or eliminate symptoms of bronchial cancer is HDR brachytherapy. Used as a palliative treatment, it aims to reduce discomfort and improve the quality of life of the patients [24,25]. The brachytherapy treatment plan can be based on standard 1D imaging. In this case, the dose distribution is presented in virtual form along the unreal rectilinear applicator. Neither the anatomical conditions of the patient 
nor the curvature of the applicator are included in the dose distribution. The treatment plan can also be based on $2 \mathrm{D}$ or $3 \mathrm{D}$ images. If the images are $2 \mathrm{D}$, then the curvature of the applicator is taken into account. 2D imaging does not provide complete information about the dose in the risk area or critical organs [16]. A treatment plan based on 3D imaging contains information on the spatial distribution of the dose in the patient's body. When planning a treatment, we have real information about the position of the applicator, target and OAR on CT images. According to the recommendations for brachytherapy of lung cancer, by 2016 any treatment should have been based on a standard treatment plan with dose specification at a distance of $1 \mathrm{~cm}$ from the applicator axis [2 $4,26,27,28,29,30,31,32,33,34]$. It is only in the 2016 guidelines published by the American Brachytherapy Society (ABS) regarding brachytherapy of bronchial cancer that three-dimensional imaging is recommended for diagnostic purposes and in a computerized TPS. It is also recommended, in connection with the use of CT, to define the therapeutic area above standard dose specification points [35]. The guidelines from 2017 for brachytherapy of bronchial cancer show that 3D planning using two bronchial applicators allows a more conformal dose distribution in the target and greater OAR protection than when using a single bronchial applicator [36]. Based on 3D images, it is possible to obtain a conformal distribution of the isodose against the target with simultaneous control of the treatment toxicity $[37,38]$. One applicator is used as standard in TP in EB [39,40]. The possibility of using several applicators during the creation of a CP treatment plan allows enhanced protection of critical organs, while at the same time providing larger coverage of the PTV. Based on the available data it is recommended to perform 3D image based planning using at least two applicators [41]. As reported in the literature, it is very common that three-dimensional imaging is performed after the application of catheters has been completed, but it is then used only to verify the applicator's/applicators' position $[42,43]$. The cited literature also analyzed the position of the catheters against each other and anatomy during the patient's respiratory movement. The difference for $V_{100}$ was $2.3 \%$ [44]

Analyzing the graphs showing the comparison of both methods (Fig. 2) for the risk area, one can observe that in the $\mathrm{CP}$ method $50 \%$ of the middle results are much higher in the graph than it is in the case of the SP method, which indicates a much larger PTV acquisition in the reference method in the CP method. It could be anticipated that with higher scores in the region of the PTV, the organs at risk and critical structures will also receive significantly higher doses in the $\mathrm{CP}$ method. Increasing the dose in OAR could contribute to an increase in the treatment toxicity. To assess the toxicity of treatment, doses were analyzed in the proposed volumes of critical organs. In all analyzed OAR volumes the doses were higher in the $\mathrm{CP}$ method than in SP. When analyzing the OAR comparison for both methods in box plots (Figs. 3-6), we can observe that the differences are much smaller than in the box plot for the PTV (Fig. 2). Based on the analysis of doses in
OAR in SP and CP methods, one can observe that critical organs received slightly higher doses in the $\mathrm{CP}$ method than in the SP method.

The dose calculation algorithm used was TG-43. This algorithm is based on water mass density. Human tissues have a different mass density than water. In the case of cancer located in the lung, the dose distribution is calculated mainly in the area of lung and critical organs (heart, esophagus, spinal cord). For the OAR in the mediastinum, for example the heart, mass density is 1.05 , which is very similar to water density (1.0), and it seems correct to treat this area like an area of homogeneous water, but in the case of the lung there are such areas as a mixture of soft tissue and air. For lung (inflated) the mass density is 0.2 in the area of lung cancer. It would be worth considering a clinical treatment plan based on TG-186 and comparing the dose distribution in the target and critical organs. TG-186 algorithms take into account the tissue mass density. Analysis of both algorithms is to be a subject of the next study on comparison of the dose distribution in the target and critical organs.

TP based on three-dimensional imaging with the dose specified for the real area of the target will contribute to a significant extension of the survival time after the treatment. In the future, doses administered to OAR should be reported to enable data collection for the determination of tolerance levels for bronchial cancer treated with HDR brachytherapy.

\section{Conclusions}

Contemporary planning in comparison with standard planning allows for a more conformal distribution of doses in the target area while limiting the toxicity of treatment. Modern TP allows one to adjust the dose distribution to the individual clinical situations and allows one to improve CTV coverage and increase doses to the OAR and overall survival as well.

The research was conducted in the Department of Brachytherapy, Subcarpathian Cancer Center, Brzozów, Poland.

\section{Disclosure}

The authors report no conflict of interest.

\section{References}

1. Fernandes G, Sucena M, Lombardia E et al. Non small cell lung cancer - comparison between clinical and pathological staging. Rev Port Pneumol 2006; 12: 337-357.

2. Skowronek J, Kubaszewska M, Kanikowski M et al. HDR endobronchial brachytherapy (HDRBT) in the management of advanced lung cancer - comparison of two different dose schedules. Radiother Oncol 2009; 93: 436-440.

3. de Aquino Gorayeb MM, Gregório MG, de Oliveira EQ et al. High-dose-rate brachytherapy in symptom palliation due to malignant endobronchial obstruction: a quantitative assessment. Brachytherapy 2013; 12: 471-478.

4. Siegel R, Ward E, Brawley O et al. Cancer statistics, 2011: the impact of eliminating socioeconomic and racial disparities on premature cancer deaths. CA Cancer J Clin 2011; 61: 212-236.

5. Charles S, Lynn T, Richard A. Lung cancer: epidemiology, etiology, and prevention. Clin Chest Med 2011; 32: 605-644. 
6. Mallick I, Sharma SC, Behera D et al. Optimization of dose and fractionation of endobronchial brachytherapy with or without external radiation in the palliative management of non-small cell lung cancer: a prospective randomized study. J Cancer Res Ther 2006; 2: 119-125.

7. Kawamura H, Ebara T, Katoh T et al. Long-term results of curative intraluminal high dose rate brachytherapy for endobronchial carcinoma. Radiat Oncol 2012; 7: 112.

8. Simon M, Argiris A, Murren JR. Progress in the therapy of small-cell lung cancer. Crit Rev Oncol Hematol 2004; 49: 119-133.

9. Aumont-le Guilcher M, Prevost B, Sunyach MP et al. Highdose-rate brachytherapy for non-small-cell lung carcinoma: a retrospective study of 226 patients. Int J Radiat Oncol Biol Phys 2011; 79: 1112-1116.

10. Ung YC, Yu E, Falkson $C$ et al. The role of high-dose-rate brachytherapy in the palliation of symptoms in patients with non-small-cell lung cancer: A systematic review. Brachytherapy 2006; 5: 189-202.

11. Stout R, Barber P, Burt P et al. Clinical and quality of life outcomes in the first United Kingdom randomized trial of endobronchial brachytherapy (intraluminal radiotherapy) vs. external beam radiotherapy in the palliative treatment of inoperable non-small cell lung cancer. Radiother Oncol 2000; 56: 323-327.

12. Allen AM, Abdelrahman N, Silvern D et al. Endobronchial brachytherapy provides excellent long-term control of recurrent granulation tissue after tracheal stenosis. Brachytherapy 2012; 11: 322-326.

13. Stephens K, Wood D. Bronchoscopic management of central airway obstruction. I Thorac Cardiovasc Surg 2000; 119: 289-296.

14. Henschke U, Hilaris B, Mahan G. Remote afterloading with intracavitary applicators. Radiology 1964; 83: 344-345.

15. Strnad V, Potter R, Kovács G. Practical handbook of brachytherapy. Uni-Med Verlag, Bremen-London-Boston 2014; 166-118.

16. Skowronek J, Piotrowski T, Ramlau R et al. The repeated use of high dose rate brachytherapy for locally recurrent lung cancer. Rep Pract Oncol Radiother 2003; 8: 127-137.

17. ICRU. Bethesda, USA: ICRU; 1997. Dose and volume specification for reporting interstitial therapy, ICRU Report 58.

18. Sawicki M. Treatment planning in brachytherapy HDR based on three-dimensional image. Computed tomography-advanced applications. InTech 2017; 2: 27-62.

19. Sawicki M, Kazalski D, Lyczek J et al. The evaluation of treatment plans in high-dose-rate endobronchial brachytherapy by utilizing 2D and 3D computed tomography imaging methods. J Contemp Brachytherapy 2014; 6: 289-292.

20. Kolkman-Deurloo K, Visser AG, Niel CG et al. Optimization of interstitial volume implants. Radiother Oncol 1994; 31: 229-239.

21. Edmundson G. Geometry based optimisation for stepping source implants Brachytherapy HDR and LDR. Proceeding Brachytherapy Meetring, 1989.

22. Ahmad F, Aletti P, Charra-Brunaud et al. Influence of dose point and inverse optimization on interstitial cervical and oropharyngeal carcinoma brachytherapy. Radiother Oncol 2004; 73: 331-337.

23. Fijuth J, Makarewicz R. Brachytherapy in the treatment of patients with lung cancer. Wspótcz Onkol 2002; 6: 37-40.

24. Celebioglu B, Gurkan OU, Erdogan S et al. High dose rate endobronchial brachytherapy effectively palliates symptoms due to inoperable lung cancer. Jpn J Clin Oncol 2002; 32: 443-448.

25 . Skowronek J. Brachytherapy in the treatment of lung cancer a valuable solution. J Contemp Brachytherapy 2015; 7: 297-311.

26. Nag S, Abitbol AA, Anderson LL et al. Consensus guidelines for high dose rate remote brachytherapy in cervical, endo- metrial, and endobronchial tumors. Int J Radiat Oncol 1993; 27: 1241-1244.

27. Van L, Pötter R. Bronchus Cancer. The GEC ESTRO handbook of brachytherapy, European Society of Therapeutic Radiology and Oncology, Brussels 2002.

28. Rodrigues G, Videtic GM, Sur R et al. Palliative thoracic radiotherapy in lung cancer: an American Society for Radiation Oncology evidence-based clinical practice guideline. Pract Radiat Oncol 2011; 1: 60-71.

29. Reveiz L, Rueda J, Cardona AF. Palliative endobronchial brachytherapy for non-small cell lung cancer. Cochrane Database Syst Rev 2012; 12: CD004284.

30. Skowronek J, Piorunek T, Kanikowski M et al. Definitive high-dose-rate endobronchial brachytherapy of bronchial stump for lung cancer after surgery. Brachytherapy 2013; 12: 560-566.

31. Mantz C, Dosoretz D, Rubenstein J et al. Endobronchial brachytherapy and optimization of local disease control in medically inoperable non-small cell lung carcinoma: a matched-pair analysis. Brachytherapy 2004; 3: 183-190.

32. Kobayashi K, Murakami N, Inaba K et al. Dose reconstruction technique using non-rigid registration to evaluate spatial correspondence between high-dose region and late radiation toxicity: a case of tracheobronchial stenosis after external beam radiotherapy combined with endotracheal brachytherapy for tracheal cancer. J Contemp Brachytherapy 2016; 8: 156-163.

33. Murakami N, Kobayashi K, Nakamura S et al. A total EQD2 greater than 85 Gy for trachea and main bronchus D2cc being associated with severe late complications after definitive endobronchial brachytherapy. J Contemp Brachytherapy 2015; 7: 363-368.

34. Nguyen N, Timotin E, Hunter R et al. Endotracheal brachytherapy alone: An effective palliative treatment for tracheal tumors. Brachytherapy 2015; 14: 543-548.

35. Stewart A, Parashar B, Patel M et al. American Brachytherapy Society consensus guidelines for thoracic brachytherapy for lung cancer. Brachytherapy 2016; 15: 1-11.

36. Van Limbergen E, Skowronek J, Pötter R. Bronchus cancer. In: Van Limbergen E, Pötter R, Hoskin P, Baltas D (eds.). The GEC-ESTRO Handbook of Brachytherapy. $2^{\text {nd }}$ ed. DOVE platform ESTRO; Brussels 2017.

37. Tsai J, Cardarelli G, Corrao A et al. SU-FF-T-24: Comparison of Endobronchial HDR Brachytherapy Using CT Imaging and Conventional Simulator Filming. Med Phys 2005; 32: 1954.

38. Lyczek J, Kazalski D, Kowalik $€$ et al. Comparison of the GTV coverage by PTV and isodose of $90 \%$ in 2D and 3D planning during endobronchial brachytherapy in the palliative treatment of patients with advanced lung cancer. Pilot study. J Contemp Brachytherapy 2012; 4: 113-115.

39. Hennequin $C$, Tredaniel J, Chevret $S$ et al. Predictive factors for late toxicity after endobronchial brachytherapy: a multivariate analysis. Int J Radiat Oncol 1998; 42: 21-27.

40. Ladi L, Grecula J, Allen E et al. High dose rate (HDR) dual intraluminal brachytherapy catheter in treatment of bilateral endobronchial tumor progression: a case report. Chest 2015; 148.

41. Skowronek J. Brachytherapy in the treatment of lung cancer a valuable solution. J Contemp Brachytherapy 2015; 7: 297-311.

42. Senan S, Lagerwaard FJ, de Pan C et al. A CT-assisted method of dosimetry in brachytherapy of lung cancer. Rotterdam Oncological Thoracic Study Group. Radiother Oncol 2000; 55: 75-80.

43. Nomoto Y, Shouji K, Toyota S et al. High dose rate endobronchial brachytherapy using a new applicator. Radiother Oncol 1997; 45: 33-37.

44. Cavanaugh S, Vidovic A, Law T et al. Multichannel endobronchial HDR catheter respiratory motion and resultant dosimetric variation. Brachytherapy 2015; 14: S47. 